Bulgarian Journal of Physics vol. 49 (2022) 5-20

\title{
Mentors Matter: A Tribute to David J. Rowe
}

\author{
J.P. Draayer \\ Department of Physics and Astronomy, Louisiana State University, \\ Baton Rouge, LA 70803 USA
}

Received 24 November 2021

doi: https://doi.org/10.55318/bgjp.2022.49.1.005

\begin{abstract}
The role David Rowe played in advancing our understanding of nuclear structure has been pivotal to modernizing the theory into one that does not require the use of an effective charge concept, and one that is extensible across the chart of the nuclides. We briefly review how this flows from the early history of the field, focusing especially on David's role in helping to bridge the divide between single-particle models and collective models of nuclear structure, and how this has led to the development of a beautiful and bold algebraic framework that underpins a theory of choice for future nuclear structure studies.

Looking forward, David Rowe's work also underpins current follow-on efforts focused on paving the way for the construction of yet another bridge, one that should help to span the chasm between low-energy and high-energy nuclear physics. If successful, the latter could ultimately lead to a truly $a b$ initio framework for gaining a far broader understanding of nuclear structure, one that tracks forward from the fundamental structure of hadrons, especially protons and neutrons, and how they in turn conspire to give us atomic nuclei that in reality are the building blocks of the universe in which we live.
\end{abstract}

KEY WORDS: Single-particle model, collective model, SU(3) shell model, symplectic model, no-core shell model (NCSM), symmetry-adapted NCSM (SANCSM), symplectic effective field theory (Sp-EFT).

\section{Introduction}

Based on its history, the field of subatomic physics can be subdivided into three major time domains. The first two of these span the whole of the $20^{\text {th }}$ century, with a break point just beyond the mid- $20^{\text {th }}$ century mark, with a third one spanning the first decades of the $21^{\text {st }}$ century.

The first of these starts with the pioneering work of Ernest Rutherford [1] (England, Nobel Prize 1908) that ultimately led to the nuclear model of the atom 
that was advanced by his colleague Niels Bohr [2] (Denmark, Nobel Prize 1922) who in 1913 proffered his text-book view of the structure of the atom, and which ultimately led Niels Bohr to focus his attention on nuclear physics starting with a relatively simple liquid-drop picture of nuclei that supported vibrational as well as rotational modes and under the right conditions, even nuclear fission. This was followed by two game-changing developments, both products of the late $40 \mathrm{~s}$ and early 50s; specifically, work that built forward from that of Niels Bohr by his son Aage Bohr and Ben Mottelson (Copenhagen School, Nobel Prize 1975, shared with James Rainwater), generally known as a collective model of nuclear structure [3], and another based on the pioneering work of Maria GoeppertMayer and J. Hans D. Jensen [4] (Nobel Prize 1963, shared with Eugene Wigner) for advancing a particle-based shell-model picture of nuclei, which in its simplest form amounts to placing neutrons and protons into a three-dimensional harmonic oscillator (3D-HO) potential with a spin-orbit term added to account for the separation of levels within a given shell.

These two camps split subatomic physics around the middle of the $20^{\text {th }}$ century into two modalities: one focused on collective modes and the other on particle modes, with remnants of this divide persisting until today. However, that landscape was eclipsed by the pioneering work of J. P. (Phil) Elliott (British/Sussex School), who in the late 50s and early 60s [5] published a series of papers showing that the valence space of a many-particle, harmonic-oscillator-based shellmodel theory could be reorganized into irreducible representations (irreps) of SU(3), where the latter - with its two (Casimir) invariants, one of $2^{\text {nd }}$ order and another of $3^{\text {nd }}$ order - could be linked to the $\beta$ (prolate extension) and $\gamma$ (triaxiality geometry) of an ellipsoidal characterization of the collective model.

This game-changing event expanded into other significant activities that ultimately became known as algebraic approaches to nuclear structure, where a primary group along with its subgroups and subgroup chains - typically aligned with experimentally known phenomena - tell an interesting tale about the dynamics within such a framework. For example, perhaps the best known and most widely acclaimed of all such algebraic models is the Interacting Boson Model (IBM, boson based) of Akito Arima and Franco Iachello [6] with its primary $\mathrm{SU}(6)$ group and three subgroup chains (U(5) for vibrations, $\mathrm{SU}(3)$ for rotations, and $\mathrm{O}(6)$ for intermediate mixed mode triaxial configurations), that overlapped time-wise with the advent of the seminal work of David Rowe and George Rosensteel $[7,8]$ on the Symplectic Model $(\operatorname{Sp}(3, \mathbf{R})$, fermion based). (See the timeline presented in subsection 3.5 ahead.)

\section{Revisiting the 'Mentors Matter' Landscape}

As the beauty, simplicity and ultimate utility of the theory David Rowe helped to define and shape, building forward from the classics noted above - the most importantly among them being the work of Phil Elliott - I think it is important, as naive as it may seem, to pause briefly to proffer a list of at least some of the 


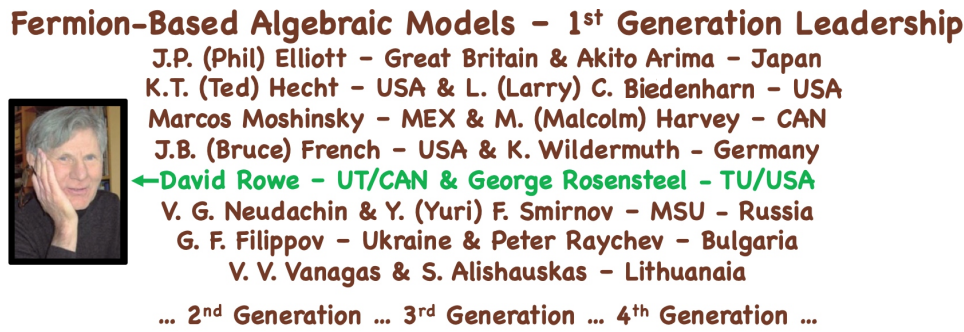

Figure 1. (Color online) Fermion-based algebraic models $-1^{\text {st }}$ generation leadership.

most prominent $1^{\text {st }}$ generation researchers (most now passed) that I intersected with and who I know played key roles individually and collectively, especially in the mentoring of students many of whom are now mentoring student of their own - in support of continuing to advance this important research agenda, see Figure 1. Prominent in this sphere of scholars, and highlighted in the figure, is David Rowe [7, 8] as well as his student George Rosensteel [8,9] - with Tulane U. in New Orleans his home institution - a friend and colleague of mine - within just an hour's drive from my home school, Louisiana State U. in Baton Rouge.

Mea culpa to those whose names should be on such a list and are not - I complied it from memory, not through a thorough vetting of who's who in the field, but rather to emphasize the depth and breath of engagement of researchers from around the world in work that is complementary and follow-on to that of Phil Elliott and as well, for the purposes of this tribute, to the work of David Rowe.

For completeness, and in special appreciation for recommendations from the (anonymous) referee of this article, I am including a short list of four of David's most pedagogical and significant single-author publications over the period of 1985-2017, see: [10-13]. The last of these, which can be downloaded from the archives (arXiv:1710.04150v1 [nucl-th] 11 Oct 2017), is highly recommended as a comprehensive review of David's life-long work that far exceeds what I have chosen to include in this short, but sincere tribute to David J. Rowe.

I believe this is how David Rowe would like to be remembered by all; especially other co-workers and mentors as well as friends via his interactions with them and their contributions to his work along the way, and through additional efforts moving forward, especially by students - who will hopefully continue this campaign and related efforts that may as well stimulate others to engage. The latter includes efforts that are focused on applications that serve to confirm and affirm the value of what was now in play and to stimulate and inspire new and some potentially very consequential next-generation nuclear physics exploits, including the trumpeting of even longer-term programs - as suggested in the abstract that David Rowe - a gentleman scholar-scientist, who I believe with his clearly demonstrated insight and focused approach to science would himself embrace and encourage, and most likely lead if he were still with us today. 
All in all, it is an honor and privilege for me to have known David Rowe throughout most of his career; and, I look forward to continuing to learn from others any gems that I may have overlooked in developing this brief tribute to David, some of which may be speculative in nature as I remain convinced there is a lot of interesting work that remains to be done by next generation young and talented follow-on researchers, all of whom would be well-advised to follow the lead of Master Mentor and Distinguished Professor David John Rowe (1936 to 8 May 2020) in their approach to scientific discovery.

\section{Symplectic Sp(3,R) Model for Nuclear Physics Studies}

So why is the symplectic $\operatorname{Sp}(3, \mathbf{R})$ concept and model so important in nuclear physics? And since the major purpose of this paper is to pay tribute to David Rowe (and appropriately, along with him, his student and life-long friend and colleague George Rosensteel, a team that I will respectfully reference going forward by simply $\mathbf{R} \& \mathbf{R}$, it is important to put everything on the top of the table, front and center, as this will allow us to take a more objective look at where things stand today in light of the discoveries made in the past as well as some missed-opportunities along the way and in so doing reiterate the theme of how important it is in the pursuit of discovery-level science to push any/all folklore aside in favor of asking penetrating questions and expecting to get deep and thoughtful responses, resisting along the way any and every temptation that may come along to yield to what at the time may seem to be an easy out, especially when the going gets tough. In keeping with the spirit of table-top talk, please note that in the literature it is also commonplace to use $\operatorname{Sp}(6, \mathrm{R})$ for $\operatorname{Sp}(3, \mathbf{R})$; we use the latter as that is what the $\mathbf{R} \& \mathbf{R}$ team have chosen to call their theory, the boldface $\mathbf{R}$ being a reminder of the 3 -space vector character of the theory.

To cut to the chase regarding the above, I am convinced that the concept of using an effective charge to reproduce experimentally observed $\mathrm{B}(\mathrm{E} 2)$ transition rates cast a blight on our field that slowed progress and intercepted our gaining the deeper understanding of nuclear structure we have today - which is due by-andlarge to the focused efforts of $\mathbf{R} \& \mathbf{R}$ in helping to uncover 'simplicity within complexity' in atomic nuclei - that today it seems might even be appreciated in terms of an effective field theory for atomic nuclei, that in turn might further evolve into a constructive path forward for helping to bridge the chasm between low-energy and high-energy nuclear physics. The latter- if correct - could lead to a truly $a b$ initio theory of the structure of atomic nuclei.

I think David Rowe would have enjoyed being part of such a discussion, with a twinkle in his eyes and and a grin on his face (see Figure 1) that only he could give! In lieu of not being able to change history, my purpose in taking this particular approach to the challenge of documenting a strong tribute to the work of the David Rowe, is to spin this out and proffer a story-line that could also be framed in terms of addressing the following three points: 1) Focus on what's 
big, and the little stuff will fall into place; 2) Expose folklore early whenever possible, as doing so can lead more quickly to a better understanding of the fundamentals that are in play; and 3) Never underestimate the importance of encouraging good and smart questions from students as they are the future! In short, I hope as this story unfolds it will provide an appropriate response to the 'Why $\mathrm{Sp}(3, \mathbf{R})$ ?' question asked in the introductory sentence of this section.

\subsection{Revisiting the role of symplectic symmetry in physics}

The symplectic group concept is a mathematically sound and unique construction that is known to be useful in physics and elsewhere whenever a system displays - at least in first order - a harmonic oscillator-like behavior. In classical mechanics, for example, it is well-known that the underlying symplectic symmetry allows one to explore the full range of momentum space representations ( $\mathbf{p}$, bold face implying vector notation with components $\left.p_{i}, i=1,2,3, \ldots, N\right)$, if working in an $N$-dimensional space) and the complementary coordinate space representations (q; $\left.q_{i}, i=1,2,3, \ldots, N\right)$ under a simple constraint that Hamilton's Poisson Brackets between the p's and the q's of the system satisfies the familiar rules, namely: $\left\{p_{i}, q_{j}\right\}=\delta_{i, j}$ where $\delta_{i, j}=1$ or 0 , respectively if $i=j$ or $i \neq j$. So long as this holds, a search for a preferred representation is fully justified with the ultimate goal being to gain the simplest mathematical and physical picture possible for describing the dynamics of the system under study.

In quantum mechanics the same holds, but with the Poisson Bracket being replaced by Heisenberg's Commutation Relation between the p's and the q's, which when $i \neq j$ is still zero, but when $i=j$ it has the value $i \hbar$, where $\hbar=h / 2 \pi$ and $h$ is Plank's constant, a small but nonzero number, that recognizes the quantum nature of the system and with this the inherent uncertainties. But again, so long as these commutation relations are preserved one has the freedom to move continuously (or discretely, if desired) between any/all representations of the system, seeking to find one - just as in the classical case - that is the most natural and that yields the simplest physical picture of the system's dynamics. Of course, as in the classical case, if external interactions drive the system away from simple harmonic behavior, but these deviation(s) is(are) relatively weak compared to the natural scale of the oscillator, then the quiet assumption that is usually made is that such deviations can be handled as perturbations on top of the system's primary modes.

The totality of any/all such transformations - classical or quantum mechanical is that they will be canonical so long as they preserve the rules of the game (Hamilton's Poisson brackets or Heisenberg's commutation relations) between and among p's and the q's of the system. What this really means is that one can choose to represent such a system from a purely coordinate-based perspective or a purely momentum-based representation, or anything in between. All of this with the usual caveats added; namely, so long as the system is not subject to ex- 
ternal influences that drive it away from its simple harmonic behavior, and with a reasonable working assumption being that small departures can be managed as perturbations to the primary modes.

The above is admittedly elementary and old school physics, but worth reviewing here because it seems to apply in spades to nuclear physics, up and down the chart of the nuclides, in ways that have heretofore not be fully understood and/or appreciated. First of all, as should be clear, the symplectic concept can and should be pulled into play whenever there is a binding potential (3D-HO in the case of nuclear physics) that in low order is harmonic in nature, and secondly when deviations therefrom are thought to be (even without sure proof) manageable in a perturbative sense. Further to this point; however, if/when a subset of the interaction terms responsible for departures from simple harmonic motion can be split into two sets, one that is a symplectic-symmetry preserving and the other that is not, with the former dominating the latter, there is still a win-win situation to be had that comes to the fore at relatively low cost as one can still use the symplectic framework to gain a deeper appreciation of the internal dynamics of a system than otherwise might not have been fully appreciated and/or even thought to be possible. In nuclear physics, the latter appears to be the case, especially when deformation dominates the dynamics. We will return to this matter in subsection 3.3 and 3.4 ahead after first taking a deeper dive into the meaning of what is implied when one probes even more deeply into the 'Why $\operatorname{Sp}(3, \mathbf{R})$ ?' question - the short and simple answer being that $\operatorname{Sp}(3, \mathbf{R}$ is the dynamical symmetry of the $3 \mathrm{D}-\mathrm{HO}$ - which plays a dominant and pervasive role in determining the structure of atomic nuclei.

\subsection{Revisiting the important features of the Elliott SU(3) model}

Elliott showed that basis states for any number of nucleons placed in a single valence shell of the isotropic 3D-HO can be reorganized into irreps $(\lambda, \mu)$ of $\mathrm{SU}(3)$. And since this can be correlated with the $(\beta, \gamma)$ shape variables of the collective model, it means the basis states - when organized this way - divide the space up into a (sometimes rich, as well as large) collection of SU(3) defined shapes. (See [14] for a generic $\mathrm{U}(N)$ to $\mathrm{U}(3)$ breakout (plethysm) for a fixed number of identical particles in the $n^{\text {th }}$ shell $-n$ quanta per level within the shell - of the 3D-HO with total degeneracy $N=(n+1)(n+2) / 2$.) Each such shape has a dimension given by the dimension of its $(\lambda, \mu)$ tagged representation; namely, $\operatorname{dim}(\lambda, \mu)=[(\lambda+\mu+2)(\lambda+1)(\mu+1)] / 2$. The generators of $\mathrm{U}(3)$ (9 in number) can be reduced to those that generate $\mathrm{SU}(3)$ (8 in number) along with the generator of $\mathrm{U}(1)$ that counts the number of 'oscillator quanta' associated with that shape, where the 8 of SU(3) include the 3 angular momentum generators of the $\mathrm{SO}(3)$ subgroup of $\mathrm{SU}(3)$, plus another 5 that can be thought of as the symmetric operators responsible for shifting oscillator quanta among the three directions in coordinate space, consistent with the constraints imposed by the fixed dimensional boundaries of the $(\lambda, \mu)$ irrep. 
This appealing picture - with its simple connection to the collective model and an opportunity to build, test and prove the importance of SU(3) in garnering a fresh look into the structure of atomic nuclei, replacing efforts that had been built based on the simple shell-model picture of the late $50 \mathrm{~s}$ - spurred a host of activities, typically led by a cadre of $2^{\text {nd }}$ generation of leaders - that ultimately led to the development of various SU(3) Shell Model programs in the 70s and even on into the $80 \mathrm{~s}$, a club of which I was and still am a member. Underpinning most of these efforts was the development of a robust technology for calculating SU(3) Wigner and Racah coefficients $[15,16]$. To the delight of some and perhaps surprise of others, the applications that flowed from these efforts clearly showed that the use of basis states organized under SU(3) according to the relative importance of the $(\lambda, \mu)$ irreps of SU(3), as gauged by the size of their respective Casimir invariants, clearly showed the dominance of deformation in calculated low-lying eigenstates. Along with this, when commonly used interactions were also so organized under SU(3), it became even clearer that SU(3) was a natural symmetry of choice for nuclear structure studies going forward.

Having lauded the work of Elliott and others mentioned above, in hindsight this should not have come as much of a surprise, since after all SU(3) is the symmetry group of the 3D-HO. But that picture is a static one, meaning a snapshot in time (or a time average) of what a nucleus looks like when projected onto a particular set of basis states. It does not unveil nor does it reveal anything about the inner machinations of what is really going on inside an atomic nucleus; that is, it screens out the dynamics in favor of snap shots in time, blocking out important features regarding the system's dynamics that snap-shot projections cannot disclose. First and foremost to this discussion is the fact that none of models discussed so far - including the Elliott SU(3) Model - reproduce the experimentally measured B(E2) values between and among low-lying eigen-solutions of the system. For the most part, it seems these differences were cleverly and quickly fixed through the use of an effective charge concept to compensate for missing B(E2) strengths, where the latter ranges from sometimes small numbers but usually considerably larger ones, especially in heavy nuclear species. And this brings us to the next subsection where we focus in on the need for and relevance of symplectic $\operatorname{Sp}(3, \mathbf{R})$ symmetry - which serves to pull the dynamics back into play - as is absolutely vital to gaining a better understanding of what is really transpiring within atomic nuclei, something that David Rowe dedicated much of his mid-career activities to - focused on true fundamentals of nuclear structure.

\section{3 $\mathrm{Sp}(3, \mathbf{R})$ as the dynamical symmetry of the $3 \mathrm{D}-\mathrm{HO}$}

As noted in the title of this subsection and other places, $\operatorname{Sp}(3, \mathbf{R})$ is the dynamical symmetry group of the 3D-HO as a whole. In layman's terms, what this implies - over and above what the Elliott SU(3) Model brings forward - is an ability of the theory to accommodate vertical couplings between and among shells of 
the 3D-HO complex, not just those within a single-major shell of the oscillator. This means that the 3D-HO picture affords us an opportunity to gain a better understanding the real dynamics that is in play between and among the nucleons as well as the field(s) they share as a collective when bound together to form a nucleus. A clear signal that this is not only important but absolutely essential for gaining such an understanding, for example of B(E2) coupling strengths between low-lying states of atomic nuclei as noted above, is to appreciate that the inclusion of these inter-shell coupling eliminates the need for artificially introducing an effective charge into the theory. That is, the symplectic extension of Elliott's SU(3) theory moves one from a single-shell picture into a holistic multi-shell environment that can serve many purposes, the single most important of these being the ability to reproduce enhanced B(E2) transition strengths without a need to introduce an effective charge concept. In short, this solves the long-standing conumdrum of our not being able to account for experimentally measured B(E2) transition strengths, especially between low-lying bound states, without introducing the notion of effective charges. And as we are now learning, it should also not come as a surprise that this also helps to fix a number of other features as well, like proffer realistic values for nuclear radii, and in the specific case of ${ }^{12} \mathrm{C}$ give a truly microscopic description of the Hoyle state.

The hesitancy within the nuclear physics community to move aggressively into probing matters like this is easy to understand since the model space grows combinatorially with the addition of every shell that is added to the mix, quickly moving beyond the reach of any and all computational facilities back then and even now, rendering even the simplest of tests to check into the potential validity (or not) of making a claim that this feature has to be included regardless of the cost and/or other barriers that one may encounter along the way, subject to quick rejection and being tossed into a 'cannot be done' or 'not within reach' category - end of story! As suggested, the first of these show-stoppers was and still is a lack of sufficiently robust computational resources to straight-up challenge this bogus effective charge myth. In sum, practical considerations regarding what would be required to carry out such calculations - even without growing the model space - were formidable at best and scary, certainly for a newcomer to the field and therefore not worth putting one's career on hold for - especially when it was generally understood (and accepted) that something as simple as an effective charge seemed to address the matter quite satisfactorily and simply!

However, this did not detour a theorist like David Rowe from wondering into this territory. In fact, David, working with his then student George Rosensteel (the $\mathbf{R} \& \mathbf{R}$ team) [7,8] did take this challenge on and attacked it from an algebraic perspective. As was the case with Phil Elliott, at its simplest level the genius in what they did was their recognition of the importance and uniqueness of adding the symplectic symmetry (which is the dynamical symmetry group of the 3DHO) into these considerations. Specifically, looking at this through a rear-view mirror, $\mathbf{R} \& \mathbf{R}$ realized that the 21 generators of the symplectic group, when bro- 
ken out according to the following group-subgroup structure, $\operatorname{Sp}(3, \mathbf{R}) \supset \mathrm{U}(3) \supset$ $[\mathrm{SU}(3) \times \mathrm{U}(1)] \supset \mathrm{SO}(3)$, can be divided up as follows: 9 generators associated with $\mathrm{U}(3)$ which breaks down into 1 for $\mathrm{U}(1)$ and 8 for $\mathrm{SU}(3)$ with $\mathrm{SU}(3)$ tensor characters $(\lambda, \mu)=(0,0)$ and $(1,1)$ respectfully, leaving 12 unaccounted for, that can be further divided up into 2 sets of 6 each; namely, $62 \hbar \omega$ raising operators with SU(3) tensor character $(2,0)$ and $62 \hbar \omega$ lowering operators with SU(3) tensor character $(0,2)$. The saga then continues when one recognizes that the SU(3) in this picture can be the SU(3) configurations of the Elliott SU(3) Model with the $\mathrm{U}(1)$ keeping a running tab on the number of 3D-HO quanta involved in the construction of any specific Elliott $\operatorname{SU}(3)(\lambda, \mu)$ configuration, and the 8 generators of SU(3) itself determining the internal (single-shell, $0 \hbar \omega$ ) dynamics that a specific $(\lambda, \mu)$ configuration brings to the party, and that an application of any of the symplectic $(0,2)$ lowering operators destroys.

Problem solved, or so it seemed! Given the above, it should be clear that an application of such a strategy seemed to divide the entire shell-model space up into a collection of Elliott-like SU(3) bandhead configurations (meaning an application of any of the symplectic $(0,2)$ lowering operators to any bandhead destroys it) plus the addition of any number of $(\lambda, \mu)=(2,0)$ excitations on top of a bandhead which can be expanded into an infinite-dimensional symplectic tower as follows: $(\lambda, \mu) \times[(2,0) \times(2,0) \times \cdots]$. And further, it should also be clear from these considerations that the collective of all such Elliott SU(3) bandhead configurations, together with their respective symplectic towers spans the entire shell-model space that is available to any nucleus. (We will return to a fuller discussion of this matter in subsection 3.5 below.) After all, as noted above, the objective of any symplectic theory is to find the 'simplest and best representation of the dynamics', minimizing complexities - with the only caveat being that the transformation had to be canonical and unitary.

\subsection{A simple caricature of the $\mathrm{Sp}(3, \mathbf{R})$ symplectic model}

Against this background we proffer the following notional picture for capturing the essence of the dynamics of a complex nuclear (many-body) system; specifically, that any 'nuclear system' can be factorized into two parts: The first part is a 'Fermion Core' comprised of a fixed number of protons and neutrons $A(Z, N)$ or sub-clusters thereof - that fill the lowest shells of an appropriately scaled 3D-HO, which in turn serves to define a starting set of SU(3) bandheads, all free of spurious symplectic excitations.

To this we add a second part; namely, a 'Boson Cloud' of S and D 'drones' each having SU(3) tensor character $(2,0)$ and if/when called into action come equipped with a $2 \hbar \omega$ 'energy pack' - which, to push this analogy - form a 'standing army' of warriors ready to be deployed, to any of the bandheads, and as noted in the last paragraph of the prior section, subject only to an externally imposed constraint that the number can be controlled to be less than $N_{\max }^{(\lambda, \mu)}$ and 
where the sum of all these does not exceed a total $N_{\max }$ cutoff.

A caution: Do not fall into the trap of thinking the $\mathrm{S}$ and $\mathrm{D}$ 'drones' of the above picture are in some way related to the s and d bosons of the IBM Model. The latter - are typically constrained to a be active within a single shell - not between shells - and therefore are $0 \hbar \omega$ operators within a 3D-HO framework, while the S and $\mathrm{D}$ add/subtract $2 \hbar \omega$ to the system, $\mathrm{S}$ carrying no angular momentum $(L=0)$ and D carrying two units $(L=2)$, etc. (The conjugate of these are $-2 \hbar \omega$ lowering operators). These $\mathrm{S}$ and $\mathrm{D}$ operators do not close under commutation, they consist of 6 (raising) +6 (lowering) $=12$ operators of a total of 21 generators of the symplectic group, the other 9 being those of the $\mathrm{U}(3) \supset[\mathrm{SU}(3) \times \mathrm{U}(1)]$ $\supset \mathrm{SO}(3)$ subgroups of the symplectic group as discussed above.

In light of this picturesque characterization of the $\operatorname{Sp}(3, \mathbf{R})$ model, and in anticipation of section 3.5 ahead where we look into the embedding of a collection of $\mathrm{Sp}(3, \mathbf{R})$ irreps into a comprehensive SA-NCSM framework, we pause here to reiterate an important feature of this conceptualization of the $\operatorname{Sp}(3, \mathbf{R})$ theory. Specifically, the number of 'drones' allowed could in principle range from 0 to $\infty$, however practical considerations require that this be limited to some finite number. (After all, the army of 'drones' are commissioned and empowered by the nucleon collective - the $A(Z, N)$ system of nucleons called a nucleus - that has only a certain amount of energy that it can share/spare before the $A(Z, N)$ collective (i.e., the nucleus) itself falls apart!) The NCSM provides for a resolution of this matter by setting a single $N_{\max }$ cutoff. But as noted above, within the $\operatorname{Sp}(3, \mathbf{R})$ framework one can set a bandhead imposed limit; namely, $N_{\max }^{(\lambda, \mu)}$ where the sum over all of these would naturally fall under some total $N_{\max }$ cutoff as is done with the NCSM framework. This special feature, which is unique to the $\operatorname{Sp}(3, \mathbf{R})$ framework is an important consideration because it means one could in principle curtail the vertical extension of less-favored bandheads - or even set an upper bound regarding their complete elimination in favor of morefavored bandheads if the master program was programmed to make such a decision on the fly - to gain faster convergence to key experimentally determined features, and in so doing throttle down the overall explosive growth of the model space which could and would ultimately extend the overall reach of the $(3, \mathbf{R})$ concept into the domain of heavier nuclear species.

To conclude this discussion, we proffer another further observation: This emerging picture pushes us towards a symplectic effective field theory (SpEFT) concept for atomic nuclei. This is the thesis topic of one of my current graduate students; namely, David Kekejian [17] who came to LSU for graduate study from Armenia in 2017, and who has been and continues to be tremendous support in the formulation of this concept, proving any/all basic tenants the theory as well as helping with an articulation of the accompanying story-line, including preparation of this manuscript. David is set to defend his PhD thesis in the Fall 2021, with an official graduation date from LSU set for the Spring 2022. It is for this/these reason/s that we (I, in this case) need to await any further pub- 
lic discussion of this matter until the official acceptance/completion of David Kekejian's thesis project is in place and officially recorded (published).

Suffice it to say - for present purposes - that this notion of a 'Boson Cloud' of electromagnetic origin (driven forward by the dominance of monopole and quadrupole modes within a QED framework) is really driven forward by a full appreciation for what the dominance of a quadratically defined harmonic oscillator shaped potential - that in first order serves to bind the nucleons of a nucleus into stable configurations - yields as a rational characterization of the dynamics that is in play inside a nucleus. To state this even more sharply, the real role of the 'Boson Cloud' is to simply allow the nucleons to explore larger regions of configuration space to see if there might be other new configurations that lie lower in energy than those associated with only the initial ('Fermion Core') representations. And - as is addressed in David Kekejian's thesis - this seems to suggest a potential path forward (an effective field theory) that may in some way provide for more direct link to the strong QCD-driven interaction with a gluon field that binds quarks into clusters of three for nucleons or two quarks for pions, etc. While this is a path not yet trodden, we find it to be a tantalizing prospect at least to members within the LSU nuclear theory team, given what we have learning to date and from the optics of our low-energy nuclear theory exploits! Remember, David Rowe was a master mentor and clever innovator - at LSU we are attempting to keep that spirit alive, and openly extend to you an invitation to join in the fun of a high-energy/low-energy (HE/LE) crazed LSU NP team!

\subsection{Embedding $\mathrm{Sp}(3, \mathbf{R})$ in the symmetry adapted NCSM}

Hopefully, with all of the above in place, readers will appreciate that the NCSM and the SA-NCSM should be seen as complementary to one another. First and foremost, both are open-shell theories, with the NCSM addressing its 'openness' by expanding the model space it uses in a democratic way which is consistent with a pre-Elliott SU(3) Model manner of carrying out many-particle shell model investigations; that is, by including any/all levels of a 3D-HO distribution of particles up to a maximum number of a total (system-wide) oscillator quanta called the NCSM's externally fixed $N_{\max }$ cutoff. The latter is above and beyond the simplest picture that places all particles in the lowest possible levels of the 3D-HO, subject only to the Pauli Exclusion Principle imposed constraint of no identical particles being allowed to occupy the same space. As noted above, this approach ensures completeness of the model space up the user specified cutoff, albeit with the accompanying need to address special requirements such as eliminating any/all spurious states from the theory, and so on.

Alongside the NCSM scheme, stands the SA-NCSM, which builds forward from the Elliott SU(3) Model, which one relies on to ensure that any/all particle antisymmetrization requirements of the Pauli Principle are taken into account at a 'Fermion Core' level. And to this one adds a particle-independent 'Boson 
Cloud' that can be contracted (down to zero on the low end) or expanded (upward to much higher $\hbar \omega$ levels than possible within the NCSM by focusing in on a subset of leading $\operatorname{Sp}(3, \mathbf{R})$ irreps, that can be known in advance to be required to ensure ultimate convergence to experimentally known features - such as enhanced low-lying B(E2) transition rates, rotational features of ground-band and excited states, rms radii in agreement with known values, and so on. Unfortunately - as noted in the previous section - while the latter is not yet actually implemented in any existing codes - the simple fact that one can regulate the growth of the model space by monitoring and assigning relative values to expanding some symplectic bandheads while contracting or even eliminating others by simply taking advantage of the $N_{\max }^{(\lambda, \mu)}$ regulator option - either manually (now in play), or automatically (currently not in play) - at the bandhead level bodes well for the SA-NCSM emerging as a theory of choice going forward.

To reiterate an important message, called out in the previous section, we note again that all of this seems to call for the implementation of AI methodologies to help automate and regulate the explosive growth challenge of shell-model spaces - that has to date plagued any and all attempts to carry out realistic, nocore/open-core shell model calculations using ab initio input for obtaining a deeper understanding of the structure of atomic nuclei. Notwithstanding any and all of the above, let me also emphasize that to the credit of many, especially the work of Phil Elliott [5], with follow-on efforts of the $\mathbf{R} \& \mathbf{R}$ team [7, 8], and as well David Rowe himself [10-13], plus a helpful hand from members of the original NCSM team [18], I respectfully suggest that little if any of the above considerations would be in play today.

Not covered here are a number of unfinished and open questions that seem to be screaming for additional attention. This will require an influx of new $4^{\text {th }}$ and even $5^{\text {th }}$ and perhaps even a $6^{\text {th }}$ generation of talented young researchers and innovators. There remains innovative, break-through science to be done! I strongly recommend that to all readers of this tribute to David Rowe; find yourself a wise mentor of David's type to see if you too could and would like to help us forge forward together in studies of this type suggested above, or if not move on in other directions that challenge you more. But whatever the choice may be; my personal hope and advice is that you don't along the way forget to enjoy your life to the fullest while having fun in a 'fast-lane' of exciting science!

\section{A Final Few Thoughts Directly from the Pen of David Rowe}

I wrap this up with a time line that spans David Rowe's career; walking in his shoes, as some of us have attempted to do - building forward from the seminal (game-changing) research work of Phil Elliott, that seems to me to be sound foundation! So while David J. Rowe - a scholar's scholar - may now be missed, he has certainly not been forgotten. His impact on us and our field lives on! 


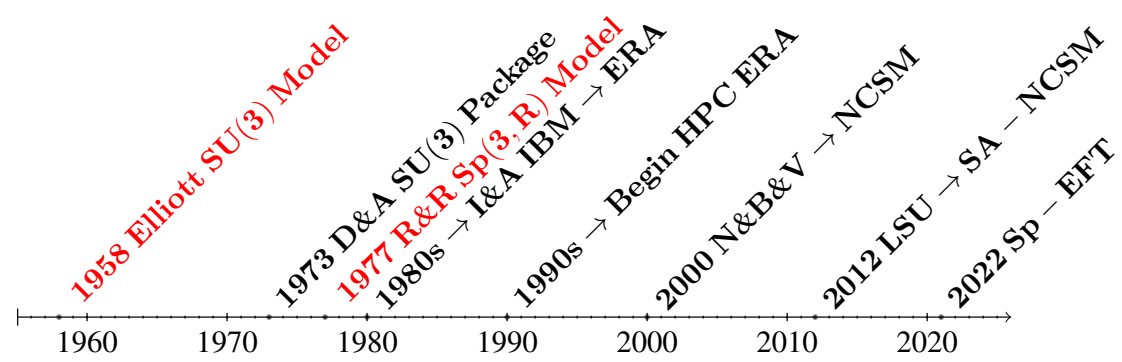

In preparing for the writing of this Tribute to David Rowe, I scanned some websites and came across a lecture that David Rowe gave in 2017. I'm inserting a couple of slides that I 'borrowed' from that lecture as I think they capture a tale that nicely reinforces David's view of the changing landscape in theoretical nuclear physics, one that he helped to effect and was now trying to understand from an implementation perspective so he could embrace it and co-own it with us, which of course was our desire! The first of these is the first slide of that lecture in which David acknowledged the 'Initial objective' of his program ('To give the collective models a microscopic foundation in shell-model terms.'), and how he seemed to be struggling with a need to deal with revised 'New objective', regarding how to deal with the emerging picture; namely, 'What does this understanding imply for the construction of optimal choices for choosing effective shell-model spaces?'.

\section{Initial objective: \\ To give the collective models a microscopic foundation in shell-model terms. \\ We find that the many nuclear models are associated with shell-model coupling schemes.}

New objective (In the light of the Draayer, Launey, Dytrych, Vary, program):

What does this understanding imply for the construction of optimal choices of effective shell-model spaces?

Major colleagues:

G.T. Rosensteel, C. Bahri, J.P. Draayer, and numerous students and postdocs (many debts to K.T. Hecht).

Figure 2. (Color online) David Rowe Lecture - Nuclear Models: Initial Objectives \& New Objectives

The latter was quite clearly a direct reference to a round-table discussion we hosted - which David Rowe and George Rosensteel - along with our LSU nuclear theory team and some others interested in 'symplectic stuff', that we hosted at LSU in 2016, during which we shared with all present where things stood in 
the development and early first case use of an early version of the SA-NCSM technology, a development that is still on-going today and that will more than likely continue well into the future. Also, note that on this slide that David called out Chairul Bahri [19], who I neglected to mention thus far in this Tribute to David Rowe, but one who rightfully deserves recognition since he was also one of my graduate students (from Indonesia), who along with Yorck Leschber and Jutta Escher (both from Germany) were early supporters of a decision to turn our attention to the development of such a framework. David's reference to Ted Hecht from the University of Michigan on this slide is is also notable since David's interactions with Ted came when I was a postdoc with Ted and it was then that he met Yoshimi Akiyama from Akito Arima's group in Tokyo and learned first hand that we (Yoshimi and I) had set ourselves to the task of developming of a generic code for calculating SU(3) Wigner and Racah Coupling Coefficents $[15,16]$ that was published in 1973 and which stood the test of time and served to underpin all SU(3)-based shell-model studies since, only this past year yielding to updated version of that old FORTRAN code into a new modernized $\mathrm{C}^{++}$what was developed over the last couple of years by Tomáš Dytrych and his Czech Republic colleagues [20].

The harmonic oscillator shell model provides the formal framework for the description of nuclear structure.

It is an exceedingly rich algebraic model with a wealth of Dynamical subgroups chains and solvable sub-models

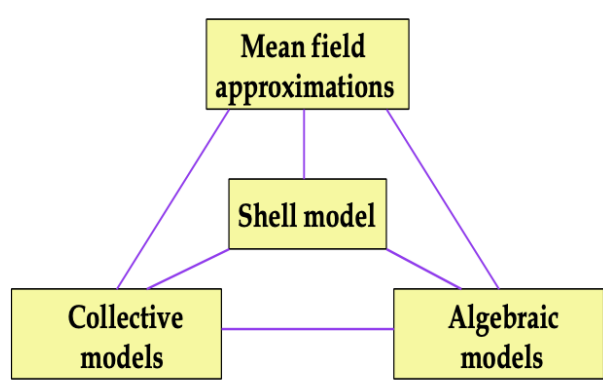

Figure 3. (Color online) David Rowe INT Lecture - Tutorial on Nuclear Models.

Figure 3 is included because this is again a succinct depiction by David himself of his view of the nuclear physics world and the central role the 'shell model' plays in this; noting in particular that 'The harmonic oscillator shell model provides the formal framework for the description of nuclear structure.' Hopefully, readers of this report gain a better appreciation for how this is indeed the case, and what it may mean for the field of nuclear physics going forward. 


\section{Conclusions}

The mystery and magic of 'symplectic simplicity' turns on the ability to factor the shell-model challenge into two features: 1) The configurations that are part of a 'Fermion Core' that looks after the 'particle parts' of the problem inclusive of any/all the associated antisymmetrization requirements since protons and neutrons are Fermions, and as well that these must be free of any/all $2 \hbar \omega$ lowering actions of the $\operatorname{Sp}(3, \mathbf{R})$ structure, in other words the 'Fermion Core' configurations should all be boson free constructions, and 2) A 'Boson Cloud' that, when added on top of the 'Fermion Core' via a direct product structure, can and must result in greater binding for the system as a whole.

And last, but by no means least, we - the community of theoretical nuclear physicists, and nuclear physicists generally - acknowledge the break-though nature of the work of J. P. (Phil) Elliott, and here, especially, the work of the late David Rowe who built forward from the work of Elliott to deliver a truly deep and lasting view for what I believe will stand as a linchpin going forward in our collective efforts to grasp the full dynamics of what is going on within atomic nuclei, and perhaps along forging a pathway for bridging the gap that still divides low-energy and high-energy nuclear physics!

\section{Acknowledgements}

The Author thanks the organizers of SDANCA-2021 for an opportunity to pay a special tribute to David J. Rowe for exciting themes surrounding the development of his science, one who stood as a pillar in our field across his entire career that spanned over 60 years. I also thank my many colleagues who have been an encouragement to me across my lifetime, especially as to how it has intersected with the work of David Rowe and his many friends and colleagues, and from time-to-time opportunities to sit around a common table to compare notes and challenge various results having fun exploring the wonders of theoretical nuclear physics and beyond. I accept responsibility for all omissions that could and probably should have been a part of this tribute to the late David J. Rowe.

This work was supported in part by the U.S. National Science Foundation (PHY1913728) and the Czech Science Foundation (16-16772S). It benefited from high performance computational resources provided by LSU (www.hpc.lsu.edu), the National Energy Research Scientific Computing Center (NERSC), a U.S. Department of Energy Office of Science User Facility operated under Contract No. DE-AC02-05CH11231, as well as the Frontera computing project at the Texas Advanced Computing Center, made possible by National Science Foundation award OAC-1818253.

Technical support of David Kekejian in preparing this tribute and for allowing use of some key (unpublished) concepts from his thesis is also acknowledged. 


\section{References}

[1] E. Rutherford, H. Geiger (1908) The charge and nature of the alpha-particle. Proceedings of the Royal Society of London. Series A, Containing Papers of a Mathematical and Physical Character 81(546) 162-173.

[2] N. Bohr (1913) I. On the constitution of atoms and molecules. The London, Edinburgh, and Dublin Philosophical Magazine and Journal of Science 26(151) 1-25.

[3] A. Bohr, B.R. Mottelson (1975) "Nuclear Structure, Vol. II: Nuclear Deformation". W.A. Benjamin, Reading, Massachusetts, USA.

[4] M.G. Mayer, J.H.D. Jensen (1955) "Elementary Theory of Nuclear Shell Structure". Wiley.

[5] J.P. Elliott (1958) Collective motion in the nuclear shell model. I. Classification schemes for states of mixed configurations. Proc. R. Soc. Lond. A 245 128-145.

[6] A. Arima, F. Iachello (1975) Collective nuclear states as representations of a SU(6) group. Phys. Rev. Lett. 35 1069-1072.

[7] G. Rosensteel, D.J. Rowe (1977) Nuclear $\operatorname{Sp}(3, \mathrm{R})$ model. Phys. Rev. Lett. 38 1014.

[8] G. Rosensteel, D.J. Rowe (1980) On the algebraic formulation of collective models. III. The symplectic shell model of collective motion. Ann. Phys. 126 343-370.

[9] G. Rosensteel, J.P. Draayer, K.J. Weeks (1984) Symplectic shell-model calculation for ${ }^{24} \mathrm{Mg}$. Nuclear Physics A 419(1) 1-12.

[10] D.J. Rowe (1985) Microscopic theory of the nuclear collective model. Rep. Prog. Phys. 48(10) 1419-1480.

[11] D.J. Rowe (1996) Dynamical symmetries of nuclear collective models. Progress in Particle and Nuclear Physics 37 265-348.

[12] D.J. Rowe (2013) The fundamental role of symmetry in nuclear models. AIP Conference Proceedings 1541(1) 104-136.

[13] D.J. Rowe (2017) The evolving many-nucleon theory of nuclear rotations. arxiv:1710.04150v1 [nucl-th].

[14] J.P. Draayer, Y. Leschber, S.C. Park, R. Lopez (1989) Representations of u(3) in u(n). Comput. Phys. Commun. 56(2) 279-290.

[15] J.P. Draayer, Yoshimi Akiyama (1973) Wigner and racah coefficients for su(3). J. Math. Phys. 14 1904-1912.

[16] Yoshimi Akiyama, J.P. Draayer (1973) A user's guide to fortran programs for wigner and racah coefficients of su(3). Comput. Phys. Commun. 5 405-415.

[17] D. Kekejian. Symplectic effective field theory. To be submitted.

[18] P. Navrátil, J.P. Vary, B.R. Barrett (2000) Properties of ${ }^{12} \mathrm{C}$ in the ab initio nuclear shell model. Phys. Rev. Lett. 84 5728-5731.

[19] C. Bahri, D.J. Rowe (2000) Su(3) quasi-dynamical symmetry as an organizational mechanism for generating nuclear rotational motions. Nucl. Phys. A 662(1) 125147.

[20] T. Dytrych, D. Langr, J.P. Draayer, K.D. Launey, D. Gazda (2021) Su3lib: A C++ library for accurate computation of Wigner and Racah coefficients of su(3). Comput. Phys. Commun. 269 108-137. 